\title{
Association between tooth impaction and odontogenic lesions: A matched case-control study
}

\author{
Shervan Shoaee ${ }^{1,2,3}$, Pegah Khazaei ${ }^{4,5}$, Fatemeh Mashhadiabbas ${ }^{6,7}$, Masoud Varshosaz ${ }^{6}$, \\ Farshad Sharifi², Hossein Hessari*4, 5
}

Received: 25 June 2017

Published: 6 Jul 2018

\begin{abstract}
Background: Impacted tooth is common in dental practice. Dentists usually remove them if they are associated with any pathologic sign or symptom. The challenge is whether to extract the asymptomatic tooth or not. This study was conducted to determine the association between developmental odontogenic cysts and tumors and impacted and unerupted teeth, if left untreated.

Methods: In this matched case-control study, 262 participants were recruited in case and control groups to evaluate the association between the presence of odontogenic cyst and tumors and unerupted and impacted teeth in patient records in the School of Dentistry, Shahid Beheshti University of Medical Sciences during 2000-2002. The controls referred to a dental radiology center for routine OPG. We also recorded the data on age, gender, the involved jaw, and type of lesion. All patients' records were reviewed by the research director and an oral pathologist separately. The variables that were matched as confounders were age, gender, and the site of the involved jaw.

Results: Results of conditional logistic regression analysis showed that the presence of odontogenic lesions was associated with impacted teeth $(\mathrm{OR}=6.9)$, and not associated with the involved jaw and unerupted teeth.
\end{abstract}

Conclusion: Impacted teeth could be considered as a potential risk factor for the presence of odontogenic lesions.

Keywords: Impacted tooth, Unerupted tooth, Odontogenic lesions

Copyright@ Iran University of Medical Sciences

Cite this article as: Shoaee Sh, Khazaei P, Mashhadiabbas F, Varshosaz M, Sharifi F, Hessari H. Association between tooth impaction and odontogenic lesions: A matched case-control study. Med J Islam Repub Iran. 2018 (6 Jul);32:57. https://doi.org/10.14196/mjiri.32.57

\section{Introduction}

An impacted tooth is a tooth that does not erupt due to malposition or lack of space in the jaw arch (1), and it is one of the most common chief complaints in those who visit dentists and maxillofacial surgeons.

In the presence of pathologic signs and symptoms, the decision to remove an impacted tooth is straightforward. However, when the impacted tooth is asymptomatic, the best decision might be a challenge $(2,3)$; some risks of intervention are alveolitis, trismus, infection, hemorrhage, dentoalveolar fractures, dental displacement, periodontal injury, injury to the nerves, and the temporomandibular joint, and in severe cases, even fracture of the mandible or the maxillary tuberosity, systemic infection, surgery

\footnotetext{
Corresponding author: Dr Hossein Hessari, h-hessari@tums.ac.ir
}

1. Otolaryngology, Head and Neck Surgery Research Center, Hazrat-e Rasool General Hospital, Iran University of Medical Sciences, Tehran, Iran.

2. Elderly Health Research Center, Endocrinology and Metabolism Research Institute, Tehran University of Medical Sciences, Tehran, Iran.

3. Non-communicable Diseases Research Center, Endocrinology and Metabolism Research Institute, Tehran University of Medical Sciences, Tehran, Iran.

4. Research Center for Caries Prevention (RCCP), Dentistry Research Institute, Tehran University of Medical Sciences, Tehran, Iran.

5. Department of Community Oral Health, School of Dentistry, Tehran University of Medical Sciences, Tehran, Iran.

6. Department of Oral and Maxillofacial Pathology, School of Dentistry, Shahid Beheshti University of Medical Science, Tehran, Iran.

7. Dental Research Center, Research Institute for Dental Sciences, Shahid Beheshti University of Medical Science, Tehran, Iran. thickened overlying bone or soft tissue, and other serious injuries (4). An impacted tooth, if not removed, may lead to misalignment, resorption of adjacent teeth, infection, odontogenic cysts, and tumors (4). In addition, the existence of pericoronal follicle adjacent to the crown of an impacted tooth is commonly associated with the formation of cysts and tumors, such as dentigerous cysts, keratocystic odontogenic tumors, and ameloblastoma, which may arise from odontogenic epithelial rests $(5,6)$.

In case of no sign or symptom, follow-up visits are suggested. Tooth extraction is preferred if the impacted tooth is accompanied by some types of pathologic lesions $(3,4$, 7). The prevalence of odontogenic lesions has been re-

$\uparrow$ What is "already known" in this topic:

Several cross sectional studies have demonstrated the association between dental follicle and odontogenic lesions.

\section{$\rightarrow$ What this article adds:}

This study found that tooth impaction is a high-risk factor for odontogenic lesions. 
ported in some studies, but it does not provide an appropriate estimate for the incidence of odontogenic lesions associated with impacted teeth $(1,8,9)$. The incidence of lesions due to dental follicles, such as odontogenic cysts and tumors, is not clear and varies from a low level to up to $59 \%$ in impacted teeth $(2,10,11)$.

This study was conducted to determine the association between developmental odontogenic cysts and tumors and impacted and unerupted teeth, if left untreated. The answer can help the dentists and patients to make clinically better decisions whether to keep or extract the tooth.

\section{Methods}

The present matched case-control study was designed based on a pilot study. Accordingly, a minimum number of 160 participants had to be recruited, 80 in the case and 80 in the control groups. However, to improve the power of the study, 131 participants were selected in each group (262 in total).

The most common odontogenic lesions (cysts and tumors) based on the textbooks of oral pathology (6) and experts' opinions (Department of Oral and Maxillofacial Pathology, Shahid Beheshti School of Dentistry) are listed in Table 1. The presence (case) or absence (control) of odontogenic lesions was considered as a dependent variable and the presence of the impacted or unerupted tooth as exposure. Age and gender were considered as background information. All patient records in the Department of Oral and Maxillofacial Pathology, Shahid Beheshti School of Dentistry during 2000-2002 were reviewed, and cases with a diagnosis of odontogenic cyst or tumor (Table 1) were selected. Accordingly, 131 participants were included in the case group.

The presence or absence of the tooth in the lesions of both groups was recorded based on radiological and pathological reports in patient records. The term "unerupted" was used when the tooth was present in the lesion and could still be expected to erupt. The term "impacted" was used when the participant was older than eruption age based on the chronologic eruption age (Table 2). For instance, a mandibular central incisor was considered as unerupted in a 7-year-old patient but impacted in a 9-yearold patient (12).

We also recorded the data of age, gender, the involved jaw, and type of lesion. All patient records were reviewed by the research director and an oral pathologist separately.

The exclusion criteria were uncertain diagnoses and missing data in the records.

The controls had no signs of odontogenic lesions (Table 1) on their radiographs based on the reports of an oral and maxillofacial radiologist. The variables that were matched

Table 1. List of common odontogenic cysts and tumors

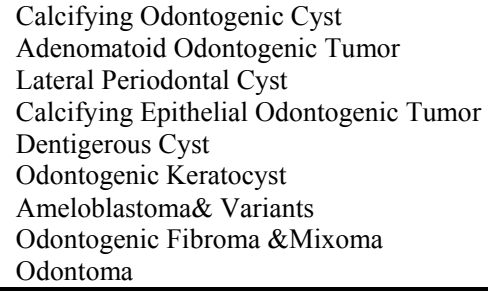

\begin{tabular}{lcc} 
Table 2. Chronology of the human dentition & \\
\hline Deciduous Dentition & Tooth & Eruption time (month) \\
\hline Maxillary & Central incisor & 7.5 \\
& Lateral incisor & 9 \\
& Cuspid & 18 \\
Mandibular & First molar & 14 \\
& Second molar & 24 \\
& Central incisor & 6 \\
& Lateral incisor & 7 \\
Permanent Dentition & Cuspid & 16 \\
Maxillary & First molar & 12 \\
& Second molar & 20 \\
& Tooth & Eruption time (year) \\
& Central incisor & $7-8$ \\
& Lateral incisor & $8-9$ \\
& Cuspid & $11-12$ \\
& First bicuspid & $10-11$ \\
& Second bicuspid & $10-12$ \\
& First molar & $6-7$ \\
Mandibular & Second molar & $12-13$ \\
& Third molar & $17-21$ \\
& Central incisor & $6-7$ \\
& Lateral incisor & $7-8$ \\
& Cuspid & $9-10$ \\
& First bicuspid & $10-12$ \\
& Second bicuspid & $11-12$ \\
& First molar & $6-7$ \\
& Second molar & $11-13$ \\
& Third molar & $17-21$ \\
\hline & &
\end{tabular}

as confounders were age, gender, and the site of the involved jaw. To match age among those younger than 25 , an exact age-matched control was performed. However, for patients over 25 years, age matching was based on the 5-year age group.

A total of 131 matched controls were selected from those who referred to a radiology center close to Shahid Beheshti Medical University for a routine OPG during the year 2003 continuously and those who referred to Departments of Orthodontics, Pedodontics, and Prosthodontics, Dental School of Shahid Beheshti University of Medical Sciences. For matching, we needed to have equal number of participants in each group; therefore, the matching process in the 2 age groups was completed by convenience sampling based on the radiological records of the patients in these 3 departments. Observers in charge of selecting the controls were blind to the presence or absence of unerupted or impacted teeth in case samples. The presence of the impacted or unerupted tooth in the matched jaw was recorded based on radiography.

Descriptive analyses were performed to obtain the frequency of odontogenic lesions. Conditional logistic regression analysis was used, and $\mathrm{p}<0.05$ was considered statistically significant. The strength of the association was expressed through odds ratio (OR) and 95\% confidence interval (CI). Data were analyzed using SPSS 17.0, Inc., Chicago, IL, USA and STATA 13 software.

\section{Results}

Of 131 cases with odontogenic lesions, $57 \%$ were male $(\mathrm{n}=74)$, and the mean age of the cases was 25 years (SD $=14.9)$. The mean age of males $(27.2, \mathrm{SD}=16.9)$ was higher than females $(22.2, \mathrm{SD}=11.2)(\mathrm{p}<0.05)$. In the case group, $73.3 \%$ of the lesions were in the mandible and $26.7 \%$ were in the maxilla. 


\begin{tabular}{|c|c|c|c|c|c|c|c|c|c|c|c|c|c|c|c|}
\hline & & 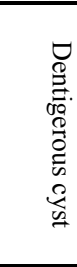 & $\stackrel{0}{\stackrel{0}{\lambda}}$ & 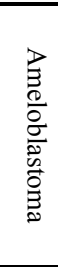 & $\begin{array}{l}\Omega \\
\dot{\gamma}\end{array}$ & $\begin{array}{l}0 \\
\stackrel{0}{0} \\
\stackrel{0}{0} \\
\stackrel{0}{0}\end{array}$ & 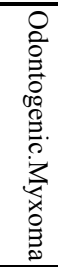 & 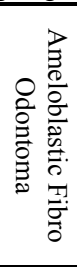 & 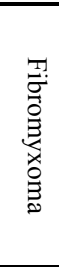 & 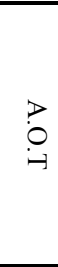 & ס) & 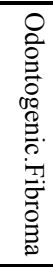 & 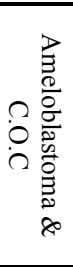 & 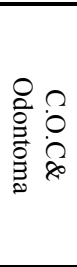 & $\stackrel{\overrightarrow{0}}{\stackrel{0}{0}}$ \\
\hline \multirow[t]{2}{*}{ Males } & $\mathrm{n}$ & 31 & 27 & 8 & 2 & 2 & 2 & 0 & 0 & 2 & 0 & 0 & 0 & 0 & 74 \\
\hline & $\%$ & 23.7 & 20.6 & 6.1 & 1.5 & 1.5 & 1.5 & 0 & 0 & 1.5 & 0 & 0 & 0 & 0 & 56.5 \\
\hline \multirow[t]{2}{*}{ Females } & $\mathrm{n}$ & 30 & 12 & 4 & 0 & 3 & 2 & 1 & 1 & 0 & 1 & 1 & 1 & 1 & 57 \\
\hline & $\%$ & 22.9 & 9.2 & 3.1 & 0 & 2.3 & 1.5 & 0.8 & 0.8 & 0 & 0.8 & 0.8 & 0.8 & 0.8 & 43.5 \\
\hline \multirow[t]{2}{*}{ Total } & $\mathrm{n}$ & 61 & 39 & 12 & 2 & 5 & 4 & 1 & 1 & 2 & 1 & 1 & 1 & 1 & 131 \\
\hline & $\%$ & 46.6 & 29.8 & 9.2 & 1.5 & 3.8 & 3.1 & 0.8 & 0.8 & 1.5 & 0.8 & 0.8 & 0.8 & 0.8 & 100 \\
\hline
\end{tabular}

Table 4. Results of conditional logistic regression analysis for the presence of odontogenic lesions

\begin{tabular}{lccccc}
\hline & OR (coefficient) & SE & P-value & Lower 95\% CI & Upper 95\% CI \\
\hline Jaw & $0.00002(-11.03)$ & 0.01757 & 0.992 & 0 & 0 \\
Impaction & $6.923(1.93)$ & 3.32484 & $<0.0001$ & 2.700 & 0.127 \\
Uneruption & $0.364(-1.01)$ & 0.19615 & 0.061 & 1.046 \\
\hline
\end{tabular}

In the case group, the first 3 common lesions were dentigerous cyst $(46.6 \%)$, odontogenic keratocyst $(29.8 \%)$, and ameloblastoma (9.2\%) (Table 3). No statistical difference was found between the 2 jaws in the frequency of impacted $\left(\chi^{2}=0.676, \mathrm{DF}=1, \mathrm{n}=131, \mathrm{p}=0.41\right)$ or unerupted teeth $\left(\chi^{2}=1.33, \mathrm{DF}=1, \mathrm{n}=131, \mathrm{p}=0.25\right)$.

Based on the conditional logistic regression analysis, the presence of odontogenic lesions was not associated with the involved jaw and unerupted teeth, but it was associated with impacted teeth $(\mathrm{OR}=6.9, \mathrm{CI}: 2.7-17.7, \mathrm{p}<0.0001)$ (Table 4).

\section{Discussion}

This study revealed that the presence of impacted teeth was associated with the presence of odontogenic lesions. The most common pathologic findings among our patients were dentigerous cysts, as mentioned in several textbooks and articles $(2,6,14,15)$.

One of the most common complaints of patients aged 16 to 30 years who visited a dentist was impacted tooth. Extraction is a straightforward decision when there is a pathologic problem or pain. The challenge is whether to leave the tooth in its place or remove it in an asymptomatic patient, especially when the tooth is near the mandibular nerve or has curved roots (2).

Some studies have reported only an association between the prevalence of odontogenic cysts and tumors and impacted teeth and concluded that pericoronal cysts and tumor development are rare. Some review studies have used this information to support the rationale for no treatment of the impacted teeth $(1,13)$. To our knowledge, no study has reported an odds ratio for the association between odontogenic lesions and impacted or unerupted teeth.

The incidence age of odontogenic cysts and tumors was 5 years higher in males than in females, which might be attributed to the earlier puberty in females. The lesions are developmental in nature and are correlated with growth. Another possible description for this difference may be gender differences in hormones influencing the growth of the epithelial lining.
It has been stated that embryonic rests in dental follicles around the impacted teeth have the potential to proliferate and develop into odontogenic lesions, which requires the presence of other unknown predisposing factors $(5,6,14)$. However, impacted teeth do not necessarily lead to odontogenic lesions and, when they do, the lesions may vary from mild to extremely severe cases (7). One study reported that the pericoronal tissue of young impacted teeth showed few pathologic changes, and there was an increased risk of pathologic changes with age (2). On the other hand, the radiographic appearance around an impacted tooth may not be a reliable indicator of the absence or presence of the disease within a dental follicle, and clinical diagnosis based only on the radiographic findings may be quite deceptive (16).

These issues bring up the following questions: Is prophylactic removal of impacted teeth, even in the absence of symptoms, necessary? What is the risk of these lesions when impacted teeth are left in place?

Some studies have shown an association between odontogenic cysts and tumors and impacted tooth $(1-3,9,10$, $15,17-20)$, but the exact risk is still unknown. The main reason is that these lesions take a long time to develop and prospective studies are hard to be conducted; therefore, most of these figures are obtained from retrospective studies or case reports.

The association between impacted teeth and odontogenic lesions and a marginal association between unerupted teeth and odontogenic lesions are highly important, which could be significantly associated if the sample size was larger. As previous studies have reported, not only impacted teeth predispose the patient to odontogenic lesions (Fig. 1, scenario A), but also space occupying odontogenic lesions may lead to tooth impaction (Fig. 1, scenario B) $(2,5,6,14)$. However, other unknown factors can cause both tooth impaction and odontogenic lesions, without any association between them (Fig. 1, scenario C).

Case-control studies are prone to antecedent-consequent bias. Nonetheless, the most probable scenario is that impacted teeth may lead to odontogenic lesions (Fig. 1, scenario A) due to following reasons: 


\begin{tabular}{|c|c|c|c|c|c|}
\hline Tooth Impaction & $\underset{\text { Scenario A }}{\longrightarrow}$ Odontogenic lesions & & Odontogenic lesions & $\overrightarrow{\text { Scenario } B}$ & Tooth Impaction \\
\hline & Tooth Impaction & $\begin{array}{l}\text { X factors } \\
\text { Scenario } \mathbf{C}\end{array}$ & $\vec{P}$ & genic lesions & \\
\hline
\end{tabular}

1. Impacted teeth can entrap dental follicles $(6,14)$.

2. The distribution of epidermal growth factor receptors in pericoronal follicles of impacted teeth can affect the progress of odontogenic lesions (5).

3. If the presence of the tooth in the dental socket per se is responsible for the lesion without predisposing factors, such as impaction, we should expect to see a higher incidence of unerupted teeth (teeth that are still in the eruption phase) in the case group than in the control group; however, the results showed the opposite, i.e., more impacted teeth were seen in cases and more unerupted teeth in controls.

4. It seems unlikely to have a lesion so large that could prevent the eruption of a tooth for years and still remain clinically undiagnosed.

The above reasons indicate that a greater part of the OR in the present study was perhaps caused by impacted teeth predisposing to odontogenic lesions. In other words, we found that the risk of odontogenic lesions increased in the presence of impacted teeth. Also, it should be taken into account that impacted teeth are risk factors and do not necessarily coexist with all lesions.

\section{Conclusion}

The probability of developing odontogenic lesions is higher in individuals with impacted teeth.

There is an inverse marginal association between odontogenic lesions and unerupted teeth.

It seems that females with tooth impaction are prone to odontogenic lesions at younger ages compared to males.

When deciding whether to extract an impacted tooth or not, the risk of odontogenic lesions, in addition to other factors, should be considered.

It seems that epidemiological indices are missing in the literature related to the risk of odontogenic lesions in the presence of impacted teeth.

\section{Conflict of Interests}

The authors declare that they have no competing interests.

\section{References}

1. Al-Khateeb TH, Bataineh AB. Pathology associated with impacted mandibular third molars in a group of Jordanians. J Oral Maxillofac Surg. 2006;64(11):1598-602.

2. Curran AE, Damm DD, Drummond JF. Pathologically significant pericoronal lesions in adults: Histopathologic evaluation. J Oral Maxillofac Surg. 2002;60(6):613-7.

3. Adaki SR, Yashodadevi B, Sujatha S, Santana N, Rakesh N, Adaki R. Incidence of cystic changes in impacted lower third molar. Indian $\mathrm{J}$ Dent Res. 2013;24(2):183.

4. Ghaeminia H, Perry J, Nienhuijs MEL, Toedtling V, Tummers M, Hoppenreijs TJM, et al. Surgical removal versus retention for the management of asymptomatic disease-free impacted wisdom teeth. Cochrane Database Syst Rev. 2016;(8):CD003879..
5. da Silva Baumgart C, da Silva Lauxen I, Sant'Anna Filho M, de Quadros OF. Epidermal growth factor receptor distribution in pericoronal follicles: relationship with the origin of odontogenic cysts and tumors. Oral Surgery, Oral Medicine, Oral Pathology, Oral Radiology, and Endodontology. 2007;103(2):240-5.

6. Rajendran R. Shafer'S Textbook of Oral Pathology (6Th Edition): Elsevier (A Divisionof Reed Elsevier India Pvt. Limited); 2009.

7. Peterson LJ. Rationale for removing impacted teeth: when to extract or not to extract. J Am Dent Assoc. 1992;123(7):198-204.

8. Van Der Sanden WJ, Mettes DG, Plasschaert AJ, Mulder J, Verdonschot EH. The influence of providing a clinical practice guideline on dental students' decision making. Eur J Dent Educ. 2004;8(1):1-6.

9. Van Der Sanden WJ, Mettes DG, Plasschaert AJ, Grol RP, Mulder J, Verdonschot EH. Effectiveness of clinical practice guideline implementation on lower third molar management in improving clinical decision-making: a randomized controlled trial. Eur J Oral Sci. 2005;113(5):349-54.

10. da Rosa FM, Oliveira MG, da Silva VP, Rados PV. Relationship between the positions of impacted third molars and the presence of dentigerous cysts. Gen Dent. 2015:43.

11. Kotrashetti VS, Kale AD, Bhalaerao SS, Hallikeremath SR. Histopathologic changes in soft tissue associated with radiographically normal impacted third molars. Indian J Dent Res. 2010;21(3):385.

12. Dean JA. McDonald and Avery's Dentistry for the Child and Adolescent: Elsevier; 2015.

13. Osunde O, Saheeb B, Bassey G. Indications and risk factors for complications of lower third molar surgery in a Nigerian teaching hospital. Annals of medical and health sciences research. 2014;4(6):938-42.

14. Neville BW, Damm DD, Chi AC, Allen CM. Oral and Maxillofacial Pathology: Elsevier; 2015.

15. Rakprasitkul S. Pathologic changes in the pericoronal tissues of unerupted third molars. Quintessence Int. 2001;32(8).

16. Kaushal N. Is radiographic appearance a reliable indicator for the absence or presence of pathology in impacted third molars? Indian J Dent Res. 2012;23(2):298.

17. Cillo JE, Ellis E, Kessler HP. Pericoronal squamous odontogenic tumor associated with an impacted mandibular third molar: a case report. J Oral Maxillofac Surg. 2005;63(3):413-6.

18. Daniels JSM. Central odontogenic fibroma of mandible: a case report and review of the literature. Oral Surgery, Oral Medicine, Oral Pathology, Oral Radiology, and Endodontology. 2004;98(3):295-300.

19. Campbell JH. Pathology associated with the third molar. Oral Maxillofac Surg Clin North Am. 2013;25(1):1-10.

20. Krithika C, Vardhan BH, Saraswathy K, Koteeswaran D, Sivapathasundaram B. Radiolucency in the anterior maxilla associated with an impacted tooth. Oral Surgery, Oral Medicine, Oral Pathol Oral Radiol Endodontol. 2007;103(2):164-8. 